\title{
Simultaneous Nasal Cavity and Brain Metastases from Primary Colon Adenocarcinoma
}

\section{Conill $C^{1}$, Vargas $A^{1}$, Capurro $S^{2}$ and Olondo $M^{2}$}

${ }^{1}$ Department of Radiation Oncology, Institute of Haematology and Oncology, Hospital Clinic Institut d'Investigacions Biomèdiques, University of Barcelona, Barcelona, Spain ${ }^{2}$ Department of Radiology, Hospital Clínic, Institut d'Investigacions Biomèdiques University of Barcelona, Barcelona, Spain

\begin{abstract}
Metastatic tumours to the nasal sinus are an exceedingly rare event. We describe a case of metastases to the nasal sinus simultaneously with brain metastases from a colonic adenocarcinoma. An 85-year-old man came to our hospital presenting epistaxis and headache. Diagnosis of the biopsy specimen showed it was a metastasis of the previously managed tumour. The patient underwent palliative radiotherapy.
\end{abstract}

Keywords: Nasal cavity; Rinosinusal tract; Brain metastases; Metastases from colonic adenocarcinoma; Radiotherapy

\section{Introduction}

Reports of cases of metastases to the nasal cavity and paranasal sinuses are rare. Up to now only seven cases of antral metastases from carcinoma of the gastrointestinal tract have been published, and only four patients with metastases to the paranasal sinus from colonic mucinous adenocarcinoma [1-7]. Even more rare is the simultaneous metastases to the sinonasal tract with intracranial spread [8]. We describe the clinical outcome of a patient with simultaneous metastases in the nasal cavity and in the brain from a colonic cancer.

\section{Case Report}

An 85-year-old man, with a previous colonic adenocarcinoma treated with a right hemicolectomy. The pathological specimen confirmed to be a colonic adenocarcinoma without node involvement (T2N0). Nine months later, the patient developed a pathological Carcinoembryonic antigen and thoracic Computed Tomography (CT) detected pulmonary metastases. At that time seven cycles of chemotherapy with capecitabine was started with stabilization of pulmonary disease. Eleven months later the patient presented nasal obstruction, epistaxis, headache, hearing and vision loss. The rhinoscopy showed a bleeding intranasal mass. A biopsy was performed and the histological diagnosis was metastasis from the previously managed tumour (colonic adenocarcinoma). The cranial CT scan revealed a nasal mass with etmoidal bone destruction and extension to this sinus. Multiple haemorrhagic supra and infratentorial metastases was also found (right cerebellum, right mesencephalic peduncle and left temporal lobe) (Figure 1).

The patient underwent palliative radiotherapy to the whole brain including the nasal tumour. CT planning dosimetry was performed and two parallel and opposed conformed fields were planned with 6 $\mathrm{Mv}$ photons. The total dose was $30 \mathrm{~Gy}$ in 10 daily fractions. At $21 \mathrm{~Gy}$ cumulated dose the nasal bleeding stopped although neurological deficits did not improve. The patient was asymptomatic from the initial headache, nasal bleeding and obstruction for two months after treatment but died due to a complicated pneumonia with pleural empyema.

\section{Discussion}

Malignant lesions arising from the epithelium of the nasal cavity are relatively rare [9]. The majority are squamous cell carcinomas derived from surface epithelium. Smaller percentages are glandular tumours apparently arising from submucosal glands or from respiratory epithelium. These tumours are often asymptomatic or mimic inflammatory diseases, leading to a delay in diagnosis. Common presenting symptoms of sinonasal tumours include nasal obstruction, facial pain, epistaxis, nasal drainage, and loss of smell, the same symptoms as those observed in patients with sinusitis and inflammatory nasal conditions. Metastases to the rinosinusal tract can be found from different primary tumors. Kidney is the most common site of primary tumour followed by lung and breast.

Although primary sinonasal adenocarcinoma is the most frequent tumour, metastasis from other tumor localizations should be ruled

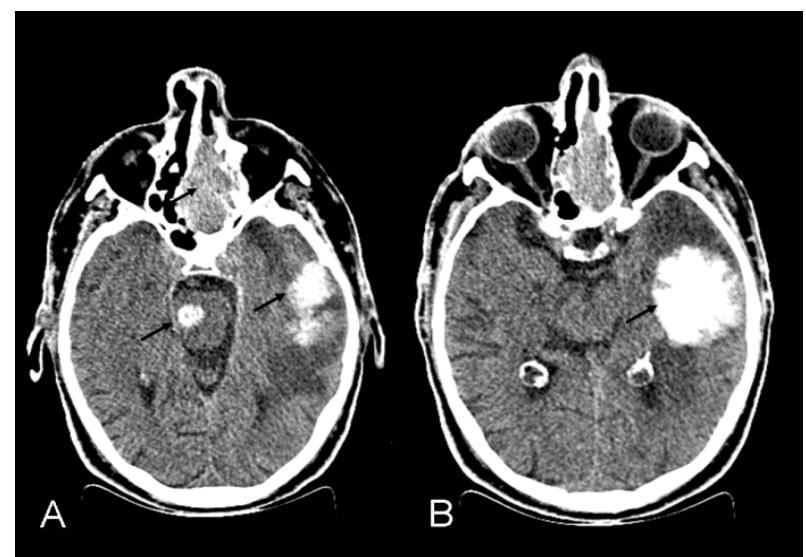

Figure 1: (A) Computed tomography (CT) without contrast shows soft tissue tumour located in left nasal cavity and left ethmoid cells with destruction of the nasal septum involving contralateral compartment. Multiple haemorrhagic intracranial metastatic lesions, with perilesional oedema were observed in the right hemi-mesencephal, and $(B)$ in the cortico-subcortical interphase of the left temporal lobe.

*Corresponding author: Conill C, Department of Radiation Oncology, Institute of Haematology and Oncology, Hospital Clinic Institut d'Investigacions Biomèdiques, University of Barcelona, Barcelona, Spain, Tel: +34 93 2275542; E-mail: cconill@clinic.ub.es

Received September 03, 2015; Accepted October 06, 2015; Published October 13,2015

Citation: Conill C, Vargas A, Capurro S, Olondo ML (2015) Simultaneous Nasal Cavity and Brain Metastases from Primary Colon Adenocarcinoma. J Clin Case Rep 5: 609. doi:10.4172/2165-7920.1000609

Copyright: ( 2015 Conill C, et al. This is an open-access article distributed under the terms of the Creative Commons Attribution License, which permits unrestricted use, distribution, and reproduction in any medium, provided the original author and source are credited. 
Citation: Conill C, Vargas A, Capurro S, Olondo ML (2015) Simultaneous Nasal Cavity and Brain Metastases from Primary Colon Adenocarcinoma. J Clin Case Rep 5: 609. doi:10.4172/2165-7920.1000609

out before a tumour is labelled as a primary of this region. When a metastasis to the sinuses is verified, the primitive tumour is generally in advanced stage and the clinical condition of the patient is compromised. Treatment is therefore palliative.

Once evidence, metastases usually involve more than one sinus cavity and are far advanced. Accurate assessment by CT provides the scientific basis for treatment approach. Treatment should be in the palliative context such as radiotherapy or chemotherapy, although surgical removal should be attempted.

Sklar [8] reported a similar case of an ethmoidal sinus lesion with extension to the skull base and into the parenchyma of the frontal lobes, with erosion of the cribiform plate. The difference with the present case was that the intracranial lesions were metastatic (supra and infratentorial) without evidence of direct extension.

No previous cases have been described presenting simultaneous metastases to the nasal tract and central nervous system. The present case represents the eighth gastrointestinal metastasic tumour to the nasal tract and the fifth case from colonic adenocarcinoma reported up to now. Radiation therapy has been successful in palliation of signs and symptoms of metastatic disease in both localizations.

\section{References}

1. Garrett MJ (1959) Metastatic tumours of the paranasal sinuses simulating primary growths. J Fac Radiol 10: 151-155.

2. Robinson D (1973) Antral metastases from carcinoma. J Laryngol Otol 87: 603-609.

3. Sera K, Yahin H, Tagashira S (1988) A case of metastatic maxillary cancer from sigmoid colon cancer Pract Oct (Kyoto) 81: 89-96

4. Shadyev KhD, DaÄniak AA, ZmoÄro AS (1989) [Metastasis of cancer of the stomach to the maxillary sinus]. Vestn Otorinolaringol : 85-86.

5. Cama E, Agostino S, Ricci R, Scarano E (2002)A rare case of metastases to the maxillary sinus from sigmoid colon adenocarcinoma. ORL J Otorhinolaryngo Relat Spec 64: 364-367.

6. Tanaka K (2006) A case of metastases to the paranasal sinus from rectal mucinous adenocarcinoma. Int J Clin Oncol 11: 64-65.

7. Conill C, Vargas M, Valduvieco I, Fernández PL, Cardesa A, et al. (2009) Metastasis to the nasal cavity from primary rectal adenocarcinoma. Clin Trans Oncol 11: 117-119.

8. Sklar EM, Pizarro JA (2003) Sinonasal intestinal-type adenocarcinoma involvement of the paranasal sinuses. AJNR Am J Neuroradiol 24: 1152-1155.

9. Granados-García M, Celis-López MA, Aguilar-Ponce JL, Villavicencio-Valencia V, Luna-Ortiz K, et al. (2006) Craniofacial resection for sinunasal tumors. Clin Transl Oncol 8: 119-123. 